\title{
Determination of the Phase Centers of Millimeter-Wave Horn Antennas Using a Holographic Interference Technique
}

\author{
Ian McAuley ${ }^{1} \cdot$ J. Anthony Murphy ${ }^{1}$. \\ Darragh McCarthy ${ }^{1} \cdot$ Marcin Gradziel $^{1}$. \\ Ronan Mahon $^{1} \cdot$ Creidhe O'Sullivan ${ }^{1} \cdot$ Neil Trappe $^{1}$
}

Received: 4 August 2015 / Accepted: 11 December 2015 /

Published online: 15 January 2016

(C) Springer Science+Business Media New York 2016

\begin{abstract}
In this paper, we discuss how a holographic interference technique can be applied in the experimental determination of the phase centers of non-standard horn antennas in the millimeter-waveband. The phase center is the point inside the horn from which the radiation appears to emanate when viewed from the far-field, and knowing its location is necessary for optimizing coupling efficiencies to quasi-optical systems. For non-standard horn designs, and other feed structures, the phase center may be difficult to reliably predict by simulation, in which case, before committing to antenna manufacture, there is a requirement for it to be determined experimentally. Although the phase center can be recovered by direct phase measurement of the far-field beam pattern, this usually involves expensive instrumentation such as a vector network analyzer for millimeter wave horn antennas. In this paper, we describe one inexpensive alternative, which is based on measuring the interference pattern in intensity between the radiation from the horn of interest and a reference beam derived from the same coherent source in an off-axis holography setup. The accuracy of the approach is improved by comparison with the interference pattern of a well-understood standard horn (such as a corrugated conical horn) in the same experimental setup. We present an example of the technique applied to a profiled smooth-walled horn antenna, which has been especially designed for cosmic microwave background (CMB) polarization experiments.
\end{abstract}

Keywords Horn antenna Phase center - Millimeter- and submillimeter-wave ·

Measurement · Holography

J. Anthony Murphy anthony.murphy@nuim.ie

1 Department of Experimental Physics, National University of Ireland Maynooth, Maynooth, Co. Kildare, Ireland 


\section{Introduction}

For non-standard millimeter-wave horn designs, and other feed structures, the phase center may be difficult to reliably predict by simulation, in which case, an experimental determination is necessary. Although the phase center can be deduced from direct phase measurements, using expensive instrumentation such as a vector network analyzer, it is also possible to recover phase information employing other methods that rely on measuring the intensity only, especially those that have been developed at other wavelengths, for example [1-5]. One such method, presented in this paper, makes use of a relatively inexpensive quasi-optical phase retrieval approach based on interference of the horn under test (HUT) with a well-understood reference beam, as in a holographic recording setup [6, 7]. In general, an interference pattern with good fringe contrast is formed when the paths of two spatially coherent beams of similar intensity intersect, provided they are derived from the same source, and the optical path difference lies within the coherence length of the radiation. The interference pattern contains information about the relative phases of the two beams, and thus, in principle, we can recover the phase curvature of the far-field beam from the millimeter-wave HUT.

A useful setup for this purpose is that used in the recording of a hologram in off-axis digital holography in the millimeter waveband, as reported in [7-9]. In [7], Mahon et al. described how an image could be reconstructed numerically from a digital hologram recorded at millimeter wavelengths in the case of a "M"-shaped mask. The application of digital holography to the terahertz band was discussed by Zhang et al. [10], while issues associated with the further development and application of holographic imaging in the terahertz band, in particular, has been investigated by a number of authors [11-13]. In Mahon et al. [7], and subsequent papers by McAuley et al. [8] and Murphy et al. [9], it was proposed that millimeter-wave digital holography could be applied to retrieve the phase center of a horn antenna without the need for direct phase measurement. However, doing this reliably proved difficult in practice in actual experimental setups [9]. In particular, the precision of the approach is compromised by any curvature of the reference beam, even though such phase curvature does not affect the sharpness of any millimeter-wave holographic images reconstructed numerically. The difficulty is that in the millimeter-wave band because of the long wavelengths involved, diffraction becomes dominant in systems of a reasonable physical size (a few 10s of centimeters). This makes it difficult to ensure the reference beam actually remains collimated, as is usually assumed in digital holography computations. Therefore, the further development of the technique, reported in $[8,9]$, necessitated improvement in the practical experimental approach, which takes the reference beam phase curvature into account.

The approach we have taken here is to relax the necessity for a plane-wave reference beam (and allow some phase curvature). Instead, what is needed is that a second interference holographic pattern is recorded with precisely the same optical setup, except that the HUT is replaced with a standard horn antenna with a well known phase center. For example, a corrugated conical horn or a waveguide probe (acting as a point-source radiator) can provide an ideal standard phase center. In this case, both of the holographic interference patterns (of the HUT and standard horn) will be affected in exactly the same way by the non-ideal aspects of the reference beam, especially the phase curvature. Thus, by analyzing the differences in the two interference patterns, we can calibrate out any phase curvature of the reference beam and recover the location of the phase center of the HUT with respect to that of the standard horn. For instance, the two recorded holographic interference patterns, although in appearance very similar, will have somewhat different fringe spacings. We can use this fringe spacing directly 
to recover the difference between the two phase center positions. Alternatively, we can perform a holographic reconstruction numerically, assuming a perfect plane-wave reference beam. Then, by removing the reference beam and the real image fields through spatial filtering (in the far field), we can recover the phases of the HUT and calibrator horn beams at the plane of the recorded hologram. Both of the beams still remain distorted in phase by the presence of the same phase curvature of the reference beam. However, by direct differencing of the two distorted phase fronts, the location of the phase center of the HUT with respect to that of the calibrator horn can be extracted.

One issue that does occur is the necessary precision in locating the phase center of a horn antenna feed coupled to a quasi-optical system. The phase center can be loosely defined as the point from which the far-field seems to radiate, as though it was a spherical wave (see early papers by Teichman and others [14-16]). However, since a horn antenna is not an isotropic radiator, its equiphase fronts are not perfectly spherical, and, in fact, the first sidelobe will be out of phase with the main beam. Alternatively, we can regard the phase center as the location of the waist of the best fit Gaussian beam to the far-field pattern (for example, see papers on the phase centers of standard horn antennas using Gaussian beam mode analysis [17-19]). The higher order Gaussian beam modes then describe the sidelobes and any other non-Gaussian structure of the beam. In fact, the coupling of the best fit Gaussian to the actual beam is generally found to vary insignificantly (by less than about $2.5 \%$ ) up to a distance of $\pm W_{0}^{2} / \lambda$ from the waist position, where $W_{0}$ is the Gaussian 1/e in amplitude beam waist radius [19], which effectively sets the tolerance required. Thus, we can regard this distance as the "depth of focus" in the sense that the phase center can be placed anywhere within this region and still effectively obtain optimum coupling to the best fit Gaussian and thus to any quasi-optical system. With these points in mind, the goal therefore is to find the best fit phase curvature over the main beam of the radiation pattern, over which the beam is approximately Gaussian in shape and, also importantly, still possesses a well-defined spherical wavefront.

In Section 2, we present the theoretical basis for the holographic interference technique, in which the reference beam is allowed to have non-zero curvature. In Section 3, we discuss, for an example non-standard horn especially designed for CMB polarization experiments, the actual process followed to extract the necessary information on the phase curvature of the beam from the recorded holographic interference pattern. We then present the experimental results for the horn phase center location. In Section 4, we discuss the issues concerning the tolerances required in the experimental measurements and also compare the experimental results with theoretical predictions. Finally, in Section 5, we draw some conclusions from the work and suggest possible future extensions to the technique.

\section{Theoretical Basis for the Holographic Technique}

An example of a useful setup for the holographic interference approach to phase center retrieval is shown in Fig. 1. The spatial coherence is provided by the use of a single mode waveguide beam splitter, to which the reference beam feed and HUT are connected via waveguide sections. The use of a Gunn diode oscillator (or any other such coherent single frequency source) provides more than sufficient coherence length for the two optical paths, with one of the beams deflected by a quasi-collimating mirror forming the reference beam. In the setup, the reference beam and the beam from the HUT intersect in the region of the scan plane, where the interference pattern is recorded by a scanning detector, as illustrated in Fig. 1. 
Then, for the dominant co-polar field, which should be co-aligned with the reference beam polarization direction, we can clearly write (assuming the Fresnel approximation) that

$$
\mathbf{E}_{H U T}=A_{H U T}(x, y, z) \exp \left(-i k\left[z+\frac{\left(x^{2}+y^{2}\right)}{2 R_{H U T}(z)}\right]+i \phi_{H U T}(x, y, z)\right) \hat{n},
$$

where $z$ represents the propagation axis for the HUT (object) beam and the plane of the page in Fig. $1 \mathrm{a}$ is assumed to be the $x-z$ plane. For a well-behaved beam pattern, we can assume that $\phi_{H U T}(x, y, z)$ is a slowly varying function of $x$ and $y$ and that the radius of curvature of the best fit spherical wavefront is described by the $R_{H U T}(z)$ term (and the field amplitude $A_{H U T}(x, y, z)$ term is real). Thus, over the main beam on a given transverse plane to the propagation $z$ direction $\phi_{H U T}(x, y, z)$ is approximately constant, and we assume the phase variation is dominated by the $R_{H U T}(z)$ term for that plane. The center of curvature for this spherical phase front is the phase center we require, assuming the hologram is recorded on a plane in the farfield of the HUT (object) beam.

If, for example, the reference beam is generated by a corrugated horn antenna (a typical standard feed at millimeter-wave or long submillimeter wavelengths), we can assume a quasiGaussian linearly polarized beam pattern, which in terms of the coordinates of the reference beam $\left(x^{\prime}, y^{\prime}, z^{\prime}\right)$ can be written in the usual complex form (for the general case [19]) as

$$
\mathbf{E}_{r e f}\left(x^{\prime}, y^{\prime}, z^{\prime}\right)=A_{r e f}\left(x^{\prime}, y^{\prime}, z^{\prime}\right) \exp \left(-i k\left[z^{\prime}+\frac{\left(x^{\prime 2}+y^{\prime 2}\right)}{2 R_{r e f}\left(z^{\prime}\right)}\right]+i \phi_{r e f}\left(z^{\prime}\right)\right) \hat{n}
$$

where $z^{\prime}$ is the direction of propagation and $\hat{n}$ is the direction of polarization. In Fig. 1a, the plane of the page is assumed to be the $x^{\prime}-z^{\prime}$ plane and the amplitude term is Gaussian in shape. The phase term $\phi_{\text {ref }}\left(z^{\prime}\right)$ is slowly varying with $z^{\prime}$ and includes the effect of the usual phase slippage associated with Gaussian beams [19], as well as any constant phase. This also includes any phase offset $k \Delta z^{\prime}$ we might choose to conveniently re-locate the origin where $z^{\prime}=0$, in order to simplify the optical analysis.

As already indicated, an issue for long-wavelength millimeter-wave systems is the difficulty (because of diffraction) of arranging that the reference beam waist is located precisely at the scan plane. Instead, here, we presume that the quasi-Gaussian reference beam actually has some curvature where the hologram is recorded. Also, if the system is perfectly aligned so that the

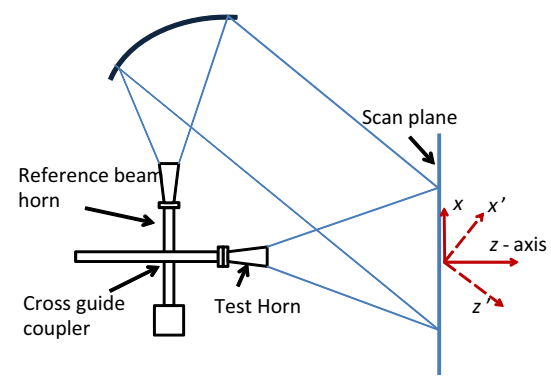

(a)

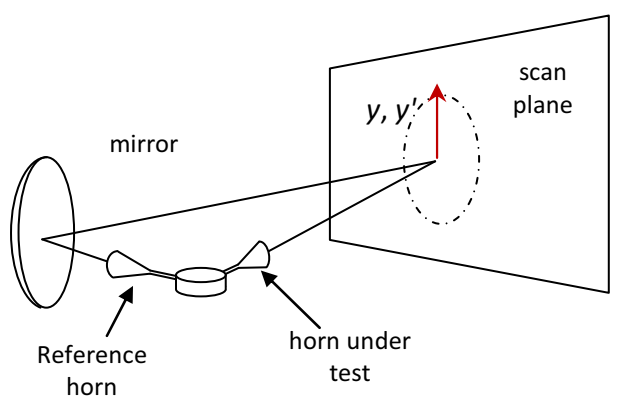

(b)

Fig. 1 Millimeter-wave holographic setup for recording interference patterns on the scan plane. a Schematic plan view and b 3-D schematic view 
optical axes of the reference and HUT beams intersect at an angle $\theta$ at a point in the center of the $(x-y)$ scan plane, we can now take this point for convenience as the common origin for both the $(x, y, z)$ and $\left(x^{\prime}, y^{\prime}, z^{\prime}\right)$ axes. This implies that we are taking $z^{\prime}=0$ and $z=0$ at the intersection point, by adding appropriate constant phase terms of the form $k \Delta z$ and $k \Delta z^{\prime}$ (and appropriately adjusting any functional forms, such as $R(z), R\left(z^{\prime}\right), W(z)$ etc., that depend on $z$ or $\left.z^{\prime}\right)$.

The interference pattern in intensity can clearly be written as

$$
\begin{aligned}
I & =\left|\mathbf{E}_{r e f}+\mathbf{E}_{H U T}\right|^{2} \\
& =\left|E_{r e f}\left(x^{\prime}, y^{\prime}, z^{\prime}\right)+E_{H U T}(x, y, z)\right|^{2}=\left|E_{r e f}\left(x^{\prime}, y^{\prime}, z^{\prime}\right)\right|^{2}+\left|E_{H U T}(x, y, z)\right|^{2} \\
& +2\left|E_{r e f}\left(x^{\prime}, y^{\prime}, z^{\prime}\right)\right|\left|E_{H U T}(x, y, z)\right| \\
& \times \cos \left[-k\left(\left(z^{\prime}-z^{\prime}\right)+\frac{\left(x^{2}+y^{2}\right)}{2 R_{H U T}(z)}-\frac{\left(x^{\prime 2}+y^{\prime 2}\right)}{2 R_{r e f}\left(z^{\prime}\right)}\right)+\phi_{H U T}(x, y, z)-\phi_{r e f}\left(x^{\prime}, y^{\prime}, z^{\prime}\right)\right],
\end{aligned}
$$

which then to a good approximation on the plane $z=0$ has the general form

$$
I(x, y)=f(x, y)+g(x, y) \cos \left(-k\left[x \sin \theta+\frac{x^{2}+y^{2}}{2 R_{0}}-\frac{x^{2} \cos ^{2} \theta+y^{2}}{2 R_{r e f}(-x \sin \theta)}\right]+\Delta \phi(x, y)\right),
$$

since in this case $x^{\prime}=x \cos \theta$ and $z^{\prime}=-x \sin \theta$, (see Fig. 1). $R_{r e f}(-x \sin \theta)$ and $R_{0}$ are the equiphase front radii of curvatures of the reference and HUT beams, respectively, over the $z=0$ plane, where $R_{\text {ref }}\left(z^{\prime}\right)=R_{\text {ref }}(-x \sin \theta)$ is a function of $x\left(\right.$ since $\left.z^{\prime}=-x \sin \theta\right)$, while $R_{0}$ is a constant for the HUT ( $z=0$ over the plane).

The terms $f(x, y), g(x, y)$ and $\Delta \phi(x, y)=\phi_{H U T}-\phi_{\text {ref }}$ are slowly varying functions of $x$ and $y$ compared to the wavelength dependent terms in the cosine function. Thus, the fringe pattern is clearly most strongly dependent on the cosine term, while for good contrast fringes $f(x, y) \approx g$ $(x, y)$ over the region where the intensity is significant. Generally, this can be arranged by varying the relative powers of the reference and HUT (object) beams using a waveguide attenuator (as, for example, illustrated in Fig. 2a). A one-dimensional cut across the interference patterns along either the $x$-direction (for a fixed value of $y=y_{m}$ ) or the $y$-direction (for a fixed value of $x=x_{n}$ ) then has the respective approximate form in the region of good fringe contrast $(f(x, y) \approx g(x, y))$ of

$$
\begin{aligned}
& I\left(x, y=y_{m}\right)=g\left(x, y_{m}\right)\left[1+\cos \left(k\left(x \sin \theta+x^{2} / 2 R_{x}(x)+\alpha_{m}\right)\right)\right], \\
& 1 / R_{x}(x)=1 / R_{0}-\cos ^{2} \theta / R_{r e f}(-x \sin \theta) \\
& \text { and } \\
& I\left(x=x_{n}, y\right)=g\left(x_{n}, y\right)\left[1+\cos \left(k\left(y^{2} / 2 R_{y}\left(x_{n}\right)+\beta_{n}\right)\right)\right] \\
& \quad 1 / R_{y}\left(x_{n}\right)=1 / R_{0}-1 / R_{r e f}\left(-x_{n} \sin \theta\right) .
\end{aligned}
$$

Such cuts could correspond to either a row or column of the recorded hologram data array, assuming it is recorded on a Cartesian grid aligned with the $x$ and $y$ axes.

Simulated examples of such patterns are shown in Figs. 2b, c. Clearly, the nulls in intensity for the two one-dimensional patterns must satisfy equations of the form:

$$
\begin{aligned}
& x \sin \theta+\frac{x^{2}}{2 R_{x}(x)}+\alpha_{m} \approx(2 \nu-1) \frac{\lambda}{2} \quad \text { for a cut in the } x \text {-direction }\left(y=y_{m} \text { constant }\right), \\
& \frac{y^{2}}{2 R_{y}\left(x_{n}\right)}+\beta_{n} \approx(2 \mu-1) \quad \frac{\lambda}{2}, \quad \text { for a cut in the } y \text {-direction }\left(x=x_{n} \text { constant }\right),
\end{aligned}
$$




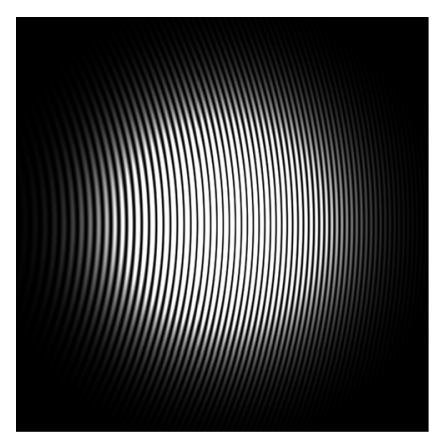

(a)

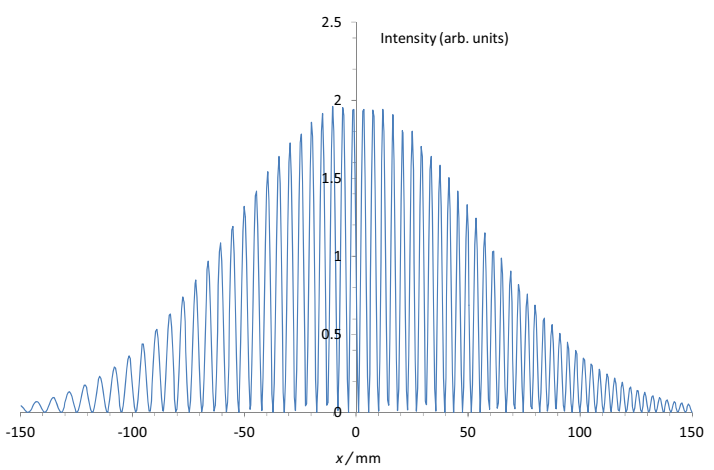

(b)

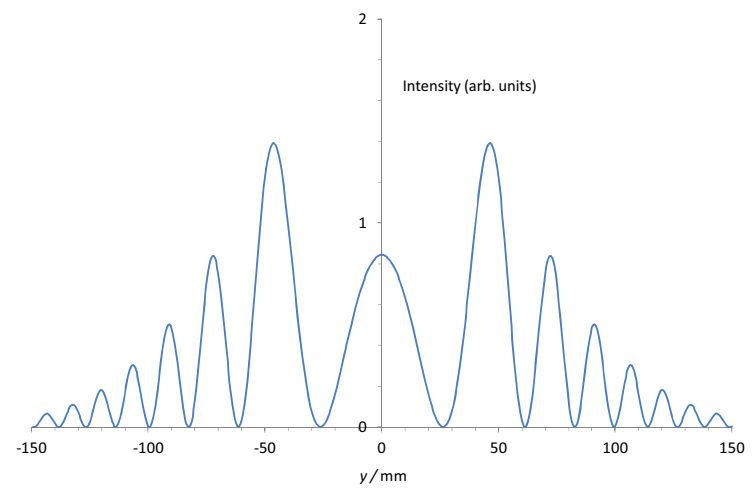

(c)

Fig. 2 a Simulated hologram with $\mathbf{b}$ a cut in the $x$-direction close to center of hologram and $\mathbf{c}$ a cut in the $y$ direction close to center of hologram

where $\mu$ and $v$ are positive integers. For a cut parallel to the $y$-axis $x=x_{n}$ is constant, allowing $R_{y}\left(x_{n}\right)$ to be calculated using these nulls in intensity. $R_{x}(x)$ is more difficult to extract as it varies with $x$ for a cut parallel to the $x$-axis ( $y$ kept constant) and, therefore for the moment, we will just extract the $R_{y}\left(x_{n}\right)$ for cuts in the $y$-direction.

If the reference beam curvature is finite, so that $1 / R_{\text {ref }} \neq 0$, clearly, $R_{0}$ cannot be recovered from a single measurement of the $R_{y}\left(x_{n}\right)$. Therefore, a second interference pattern, this time of a standard radiator with a well known phase center, can be recorded with precisely the same optical setup (the same reference beam, optical axes alignment, scan sequence, etc.). Typically, this standard phase center, as noted already, could be that of a point source (in the form of a waveguide probe), or of a well understood standard horn antenna, such as a conical corrugated horn whose radiation behavior and phase center are reliably known $[18,19]$.

Thus, if we find the $R_{y}{ }^{\prime}\left(x_{n}\right)$ for the case of the phase center of the standard horn, with $1 / R_{y}^{\prime}\left(x_{n}\right)=1 / R_{C}-1 / \mathrm{R}_{\text {ref }}\left(-x_{n} \sin \theta\right)$, where $R_{C}$ is the phase curvature of the standard antenna, then clearly (for same value of $x=x_{n}$ for the two horns), the difference in the fringe curvatures in the $y$ direction, given by

$$
\Delta\left(\frac{1}{R_{y}\left(x_{n}\right)}\right)=\frac{1}{R_{y}\left(x_{n}\right)}-\frac{1}{R_{y}^{\prime}\left(x_{n}\right)}=\frac{1}{R_{0}}-\frac{1}{R_{C}}=\Delta\left(\frac{1}{R}\right),
$$


is actually independent of the common $R_{\text {ref }}\left(x_{n}\right)$, thus allowing $R_{0}$ to be determined, and the phase center to be located.

Furthermore, if we define $\Delta z$ as the distance between the two phase centers, then clearly also $\Delta z=R_{0}-R_{C}$ and we easily rewrite this as

$$
\Delta z=-R_{0} R_{C} \Delta\left(\frac{1}{R}\right)=-\frac{R_{C}^{2} \Delta(1 / R)}{1+R_{C} \Delta(1 / R)} .
$$

In this way, we can recover the $\Delta z$, provided we have a good estimate of $R_{C}$, and a good average value for $\Delta\left(1 / R_{y}\right)$ for a large number of cuts in the $y$-direction (each with different value for $x_{n}$ ).

This approach is experimentally convenient as the phase center of the HUT antenna feed is referenced (from knowing $\Delta z$ ) to the phase center of a standard feed antenna of similar dimensions to the HUT. Thus, if the apertures of the standard horn and the HUT can in turn be referenced to a common waveguide flange (say the flange of waveguide attached to the waveguide coupler which feeds the horns) and the positions of the two horn apertures relative to this flange are accurately measured (by using a travelling microscope, for example), we can use the distance between the two phase centers $\Delta z$ to reliably locate the position of the phase center of the HUT with respect to its own aperture (without, in fact, needing to have a very precise measurement of $R_{C}$ ). In Section 3, we discuss how to recover the best average $\Delta(1 / R)$ from the measurement data for the case of an example horn antenna and hence determine the phase center location.

Alternatively, we can also use a holographic reconstruction technique to isolate the phase of the HUT beam at the scan plane (i.e., the holographic recording plane) through spatial filtering, and thus recover the phase curvatures $1 / R_{x}(x)$ and $1 / R_{y}(x)$ in Eq. 5, [7]. In this process, the digitally recorded hologram intensity pattern is multiplied by a plane-wave term travelling in the same direction of the original reference beam (with respect to the scan plane) to yield

$$
\begin{aligned}
E(x, y) & \propto \exp (i k x \sin \theta) \times I(x, y) \\
= & f(x, y) \exp (i k x \sin \theta)+\frac{g(x, y)}{2} \exp \left(2 i k x \sin \theta+i k\left[\frac{x^{2}}{2 R_{x}(x)}+\frac{y^{2}}{2 R_{y}(x)}\right]-i \Delta \phi(x, y)\right) \\
& +\frac{g(x, y)}{2} \exp \left(-i k\left[\frac{x^{2}}{2 R_{x}(x)}+\frac{y^{2}}{2 R_{y}(x)}\right]+i \Delta \phi(x, y)\right),
\end{aligned}
$$

The Fourier transform of $E(x, y)$ in turn corresponds to a numerically generated far-field pattern of the hologram illuminated by a plane-wave reference beam, which naturally separates into three diffraction orders. Spatial filtering can be used to remove the high frequency component diffraction orders (i.e., the first and second terms in Eq. 9, which represent the direct reference beam and the distorted real image of the horn, respectively) leaving the HUT (object) beam, although still with the phase distortion caused by the effect of the non-planar wavefront of the reference beam. Then, by performing an inverse Fourier transform on the spatially filtered HUT far-field beam, the distorted HUT object beam on the scan plane can be recovered

$$
E_{\text {recovered }}(x, y) \propto \frac{g(x, y)}{2} \exp \left(-i k\left[\frac{x^{2}}{2 R_{x}(x)}+\frac{y^{2}}{2 R_{y}(x)}\right]+i \Delta \phi(x, y)\right) .
$$


If we perform the same spatially filtered digital reconstruction of the corrugated calibrator horn hologram, then the difference between the two recovered phase surfaces at the hologram scan plane for the two horns, $\Delta \chi(x, y)$, is given by

$$
\begin{gathered}
\Delta \chi(x, y)=\left[\frac{x^{2}}{2 R_{x}(x, y)}+\frac{y^{2}}{2 R_{y}(x, y)}\right]-\left[\frac{x^{2}}{2 R_{x}^{\prime}(x, y)}+\frac{y^{2}}{2 R_{y}^{\prime}(x, y)}\right]+\Delta \psi \\
=\left[\frac{1}{R_{0}}-\frac{1}{R_{C}}\right] \frac{x^{2}+y^{2}}{2}+\Delta \psi=\Delta\left(\frac{1}{R}\right) \frac{x^{2}+y^{2}}{2}+\Delta \psi,
\end{gathered}
$$

where the dash refers to the standard corrugated horn as before, and $\Delta \psi$ is approximately constant (slowly varying with $x$ and $y$ ). Clearly, the difference between the recovered phase surfaces for the two horns is now independent of the reference beam phase front curvature. We can fit a spherical wavefront to this surface to recover $\Delta(1 / R)$, and then, as before, use Eq. 8 to locate the HUT phase center. In the next section, we discuss how to recover the best average phase center location using this holographic reconstruction approach for the case of an actual example non-standard horn.

\section{Recovering the Phase Center for an Example Horn Antenna}

The example HUT chosen was a non-standard smooth-walled profiled horn, especially designed for application in future generations of cosmic microwave background (CMB) polarization experiments [20]. Such experiments must be sensitive to extremely low-level polarized signals, in order to enable inflationary theories in cosmology to be further constrained, thus providing a better understanding of the formation and evolution of the Universe. In order to achieve high levels of sensitivity, it is likely that a densely packed focal plane array will be used. While corrugated horns can provide the necessary performance in terms of cross-polar power, main beam symmetry, return loss and sidelobe levels across the $10 \%$ relative bandwidths of interest for such missions (for example, the proposed CorE+ mission to the European Space Agency, ESA, [21]), they do carry thermal, mechanical, and financial penalties, which when many thousands are required, become overwhelming.

Thus, possible alternatives to corrugated horns have been investigated, and in this regard, the optimization of shaped smooth-walled horns to meet the performance demands of such missions across the band was considered as in [20]. Such smooth-walled horns can provide the necessary levels of performance in the areas of interest (e.g., cross polarization levels), but with lower penalties than those associated with corrugated horns. The design was kept as simple as possible using a linear spline-fit design consisting of six sections. By applying a genetic algorithm to the modeling of the horn using mode matching software, so as to minimize the cross-polar power, a resultant design was obtained. A profile view of the optimized horn, along with its dimensions, is given in Fig. 3a.

The horn was manufactured by Rutherford Appleton Laboratories (RAL) and measurements of the far field beam patterns in intensity were found to agree very well with predictions, verifying the horn performs extremely well across the band. At the design frequency of $100 \mathrm{GHz}$, sidelobe levels of $-50 \mathrm{~dB}$ were realized, with a highly symmetric main beam. The cross-polar power was found to be below $-25 \mathrm{~dB}$ across the $10 \%$ band, as required, with a return loss of $-20 \mathrm{~dB}$. This high level of performance, achieved using a very simple design, and without the typical Gaussian or sine-squared profiling that is normally applied to spline-fit 


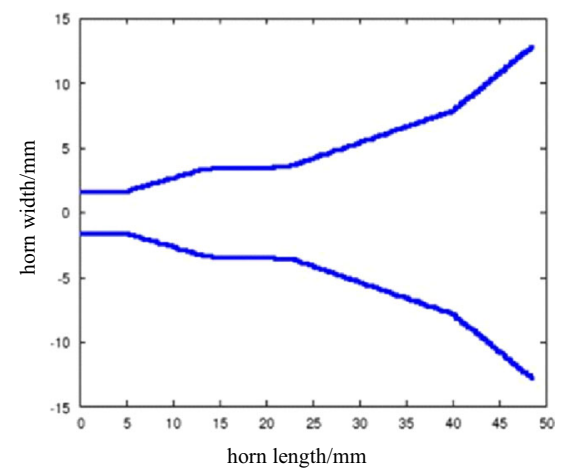

(a)

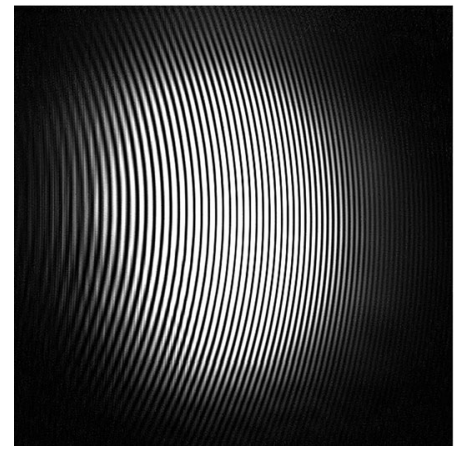

(b)

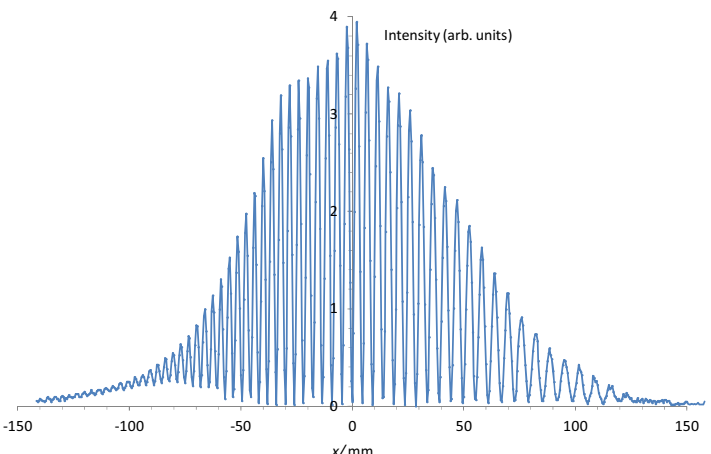

(c)

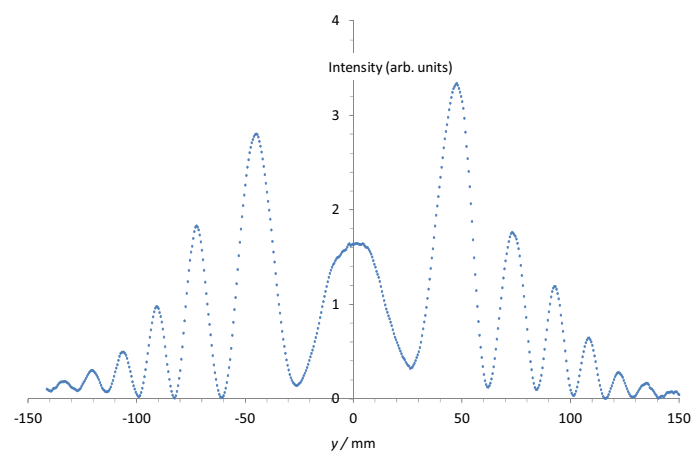

(d)

Fig. 3 a Geometry of the spline-fit smooth-walled horn for CMB polarization, as in [17]. b The recorded hologram (interference pattern) for case of the actual non-standard horn described in Section 4 and corresponding cuts in the $x$-direction $\mathbf{c}$ and $y$-direction $\mathbf{d}$ close to the center line of the image. As can be seen, there is very good agreement with Fig. 2

horns, ensured that excellent performance meeting the various optical and electromagnetic requirements could be realized relatively cheaply.

The phase center of this example horn was located experimentally using the off-axis holography setup as described above in Fig. 1. The free running Gunn oscillator operating 
at $100 \mathrm{GHz}$ (Ostek OTGW 10) provided the source of coherent radiation (line width in the $\mathrm{MHz}$ range), while the detector consisted of a waveguide probe feeding a Schottky diode rectifier (Dorado Crystal Detector CD 10). The signal source was chopped at a few kilohertz, and the detected signal recovered using a phase sensitive amplifier. The 3-dB directional coupler provides a 50:50 split of the millimeter-wave power from the Gunn oscillator. The scanner employed Zaber precision motorized XYZ stages, while the reference horn was a standard corrugated horn to produce a quasi-Gaussian reference beam. The standard calibration phase center was provided by a second well-characterized corrugated horn (both horns from TK Instruments).

The holographic interference pattern was recorded at a regular grid of points, which were separated by constant sized steps $(\Delta x, \Delta y)$ of $0.50 \mathrm{~mm}$ in the $x$ - and $y$-directions, respectively. The data were then arranged into a two-dimensional array of $600 \times 600$ intensity values $I_{m n}$, so that the $m$ th row corresponds to a traverse across the beam parallel to the $x$-axis (with $y=y_{m}$, kept constant) and the $n$th column corresponds to a traverse across the beam parallel to the $y$ axis (with $x=x_{n}$, kept constant) - consistent with the definition of the axes shown in Fig. 1 above. The recorded array of data is illustrated as an image in Fig. 3b, while an example of a row $m$ and of a column of data $n$ from the array is shown in Fig. 3c, d, respectively. In Fig. 3d, since $x=x_{n}$ is a constant for such a column $n$, the corresponding locations for the zeros $y_{\mu}$ in intensity for the $y$ direction satisfy Eq. 6 above, where $y_{ \pm \mu}$ are the locations of the two $\mu$ th zeros in intensity, with the minus sign corresponding to negative values of $y$ (assuming for the moment $y=0$ defines the symmetry axis). Thus, since $y_{-\mu}=-y_{+\mu}$ we can rewrite as

$$
\left(\Delta y_{\mu}\right)^{2}=\left(y_{\mu}-y_{-\mu}\right)^{2}=\left(2 y_{\mu}\right)^{2}=\left(8 \lambda R_{y}\right) \mu+\xi_{n},
$$

where $\xi_{n}$ is a constant for the $y$-column $\left(x=x_{n}\right)$ (see Eq. 6) and $\Delta y_{\mu}=\left(y_{\mu}-y_{-\mu}\right)$. We see that there is a linear relationship between $\left(\Delta y_{\mu}\right)^{2}$ and $\mu$, for each column $n$. Thus, we can solve for the $R_{y}\left(x_{n}\right)$ by undertaking a least squares fitting approach, which for each column involves minimizing the function

$$
K\left(R_{y}\left(x_{n}, \xi_{n}\right)\right)=\sum_{\mu=1}^{M}\left[\Delta y_{\mu}^{2}-\left(8 \lambda R_{y} \mu+\xi_{n}\right)\right]^{2},
$$

with respect to $R_{y}\left(x_{n}\right)$ and $\xi_{n}$, for the $2 M$ zeros over the main beam. Clearly, this equation does not need a knowledge of the exact location of the $x$-axis in terms of the column of data, only the $\Delta y_{\mu}$, the distance between the same order minimum on either side of $x$-axis. Finding the best-fit $R_{y}\left(x_{n}\right)$ can be automated, if there is good fringe contrast, by using software that automatically locates the local minima in a set of data (after some spatial filtering to prevent noise in the data generating false minima). The corresponding curvatures $1 / R_{y}\left(x_{n}\right)$ were plotted as a function of $x_{n}$, as illustrated in Fig. 4a. Since the incident reference beam on the measurement scan plane is at a non-normal angle of incidence, the curvatures $R_{y}\left(x_{n}\right)$ clearly vary with the column chosen as expected, as the radius of curvature of the reference beam will vary over the measurement plane and can be seen in Fig. 4a.

A second holographic interference pattern was then recorded for the standard reference corrugated horn with exactly the same optical setup as for the HUT. The corresponding $1 / R_{y}^{\prime}\left(x_{n}\right)$ were similarly determined, as described above, and are also plotted as a function of $x_{n}$ in Fig. 4a. The difference between the curvatures $\Delta\left(1 / R_{y}\right)$ as a function of position $x_{n}$, is approximately constant as can be seen in Fig. $4 \mathrm{~b}$ and as expected from Eq. 7. The average 


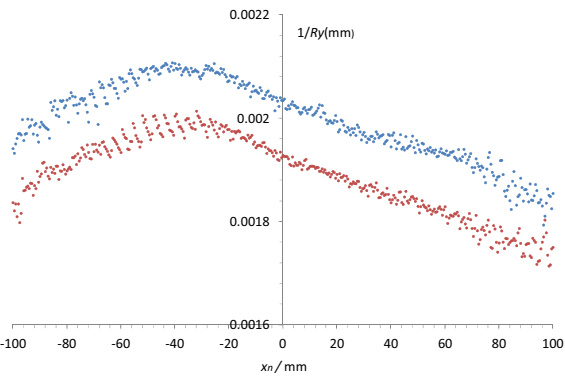

(a)

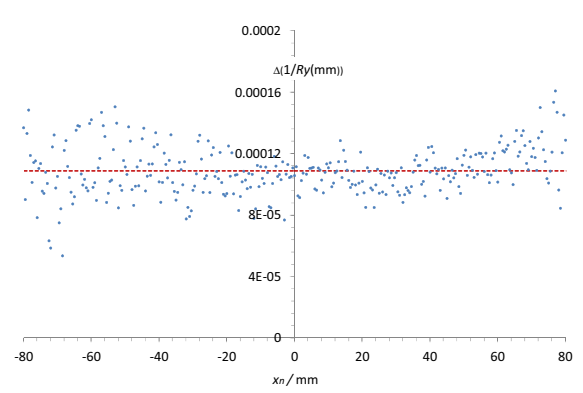

(b)

Fig. 4 a $1 / R_{y}\left(x_{n}\right)$ as a function of $x_{n}$ where the lower scatter data are for the HUT spline-fit horn antenna and the upper data are for the standard corrugated horn antenna. b The second plot shows absolute value of $\Delta(1 / R y)$

value for $\Delta\left(1 / R_{y}\right)$ near the center of the hologram (over the main beam) as shown Fig. $4 \mathrm{~b}$ is $-(1.09 \pm 0.14) \times 10^{-4} \mathrm{~mm}^{-1}$ (using the standard deviation of the estimates of $\Delta\left(1 / R_{y}\right)$ as the error). Also, as $R_{C}$ was determined to be $544 \mathrm{~mm}$, we can recover the distance between the two phase centers of the two horns $\Delta z$, using Eq. 8. This was determined to be $\Delta \mathrm{z}=(34.2 \pm 4.8)$ $\mathrm{mm}$, thus with an uncertainty of the order of the depth of focus of $7 \mathrm{~mm}$.

In the measurement setup, both horns were mounted on the same waveguide flange. The length of the spline-fit HUT feed from the flange interface with the waveguide to its aperture is $50.7 \mathrm{~mm}$ while for the corrugated horn block, including the waveguide transition, its effective aperture is at $75.0 \mathrm{~mm}$ from the flange, giving a relative distance of $24.3 \mathrm{~mm}$ between their apertures. We also have that the waist location of the corrugated horn is $6.3 \mathrm{~mm}$ behind its aperture (using a Gaussian beam mode analysis approach [9]), which is thus $18.0 \mathrm{~mm}$ in front of the location of the spline-fit horn aperture. This in turn implies, clearly, that the phase center for the spline-fit HUT must be (34.2-18.0) $\mathrm{mm} \approx 16.2 \mathrm{~mm}$ behind its own aperture, with an uncertainty of approximately $8.4 \mathrm{~mm}$ (if we add in quadrature the depth of focus to the experimental error). This implies that the beam waist region for the spline-fit horn lies in the range $(16.2 \pm 8.4) \mathrm{mm}$, approximately behind its aperture using this approach (i.e., direct comparison of the fringe spacings in the hologram interference patterns). The technique is thus sufficiently accurate to locate the phase center of the HUT with respect to the standard horn phase center.

If we apply the alternative digital holographic reconstruction approach using Eq. 9 and spatially filter the resulting high frequency terms, as described in Section 2, we can isolate the spherical phase fronts, which appear in Eq. 10, for the HUT and the standard corrugated horn. Cuts in both the $x$ - and $y$-directions through the spatially filtered phase front surfaces, recovered near the center of the hologram, are shown in Fig. 5 for the HUT. The difference between the two phase surfaces for the HUT and standard corrugated horn (as given by Eq. 11) then allows us to extract the difference in curvature between the two horns $\Delta(1 / R)$, see Fig. 6 , with the effects of the non-planar phase of the reference beam removed. The two sets of scatter data shown on the plot in Fig. 6 are for an average of a large number of cuts in the $x$ - and $y$ directions of the difference in the phase fronts (the phase difference has been normalized to zero near the axis of the HUT beam). The best fit spherical surface is displaced from the origin indicating that the origin of the axes does not quite coincide with where the beams actually intersect the scan plane. In fact, cuts in the $x$ - and the $y$-directions for this best fit spherical surface are also correspondingly displaced slightly from each other, as can be seen in Fig. 6 . 
The best-fit spherical surface has a curvature of $\Delta(1 / R)$ of $-1.17 \times 10^{-4} \mathrm{~mm}^{-1}$, from which we can recover a best fit $\Delta z=36.8 \mathrm{~mm}$ between the two phase centers, locating the phase center of the HUT at $18.7 \mathrm{~mm}$ behind its aperture, close to the previous result above obtained using the fringe spacing approach.

The best-fit curvature does vary, however, depending on the extent of the phase difference surface over which one fits the data. If larger sections of the phase difference surface are included, the curvature tends to decrease, partly because of the worse scatter in the data, but also both because the phase fronts themselves are not purely spherical and because of the fact that the $\Delta \psi$ term in Eq. 9 is slowly varying (not actually constant). Similarly, if we fit a toroid (i.e., with different radii of phase curvatures in the $x$ - and $y$-directions) to the phase difference surface over the center of the main beam, we obtain a $\Delta\left(1 / R_{x}\right)$ of $-1.36 \times 10^{-4} \mathrm{~mm}^{-1}$ and a $\Delta\left(1 / R_{y}\right)$ of $-0.97 \times 10^{-4} \mathrm{~mm}^{-1}$ giving positions for the phase center of the HUT behind its aperture of 24.6 and $11.4 \mathrm{~mm}$, respectively. If we take this variation as an approximate error bound in fitting the experimental data, and if we add the depth of focus in quadrature with this experimental error as an estimate of the full error, we obtain a result of $(18.7 \pm 9.5) \mathrm{mm}$ for the position of the phase center of behind the HUT horn aperture. This is in good agreement with the direct hologram fringe spacing method applied above, although with a larger error bound. In the next section, we discuss possible tolerancing issues associated with the experimental setup, and then compare the actual experimental results with the prediction of a theoretical model of the horn based on a mode matching approach.

\section{Tolerancing Issues}

Clearly, the accuracy of the technique depends on a high precision determination of the $\Delta(1 / R)$ for both the case of the direct estimate from the interference fringe spacings, as well as the spatially filtered holographic reconstruction approach. What is important is to recover $\Delta z$ using Eq. 8, with an error that is not greater than the focal depth of the horn. As discussed earlier in Section 1, we can take this to be about $\pm W_{0}^{2} / \lambda$, where $W_{0}$ is the equivalent waist size for the beam. This focal spread can be re-expressed in terms of the more convenient $1 / e^{2}$ semiopening angle in intensity for the far-field pattern of the horn $\theta_{0}$, given by $\lambda / \pi W_{0}$ [19], so that the range over which there is excellent coupling is given alternatively by $\pm 332 \times \lambda /\left(\theta_{0, \mathrm{deg}}\right)^{2}$, where $\theta_{0, \mathrm{deg}}$ is the $1 / e^{2}$ semi-opening angle expressed in degrees.

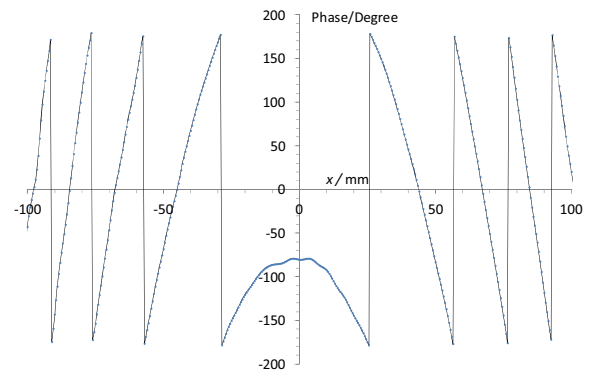

(a)

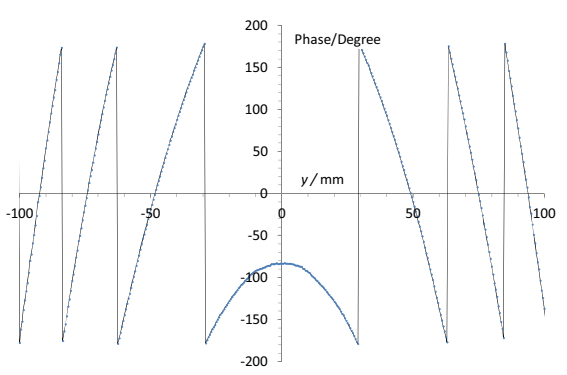

(b)

Fig. 5 Reconstructed phase front for HUT at scan plane using digital holography. a $x$-cut and $\mathbf{b} y$-cut near center of the field 
The uncertainty in the curvature difference term $\Delta(1 / R)$ should therefore satisfy the inequality

$$
\frac{\delta[\Delta z]}{\Delta z}=\left|\frac{\delta[\Delta(1 / R)]}{\Delta[1 / R]}\right|+2\left|\frac{\delta R_{C}}{R_{C}}\right| \leq \frac{332}{\theta_{0, \mathrm{deg}}^{2}}\left(\frac{\lambda}{\Delta z}\right),
$$

for a technique to be sufficiently accurate to be useful. For a typical millimeter-wave horn $\theta_{0}=12^{\circ}$ in radius at the $-8.7 \mathrm{~dB}$ level, the depth of focus is about $\pm 2.3 \lambda$, whereas for narrower beam horns, this becomes several wavelengths, which should be taken into account in assessing the usefulness of the approach.

One issue in a practical setup is that it may not also be possible to easily arrange that the optical axes of the reference and object beams intersect precisely at the scan plane (since the beams are wide there), and so they may be offset from each other in the $x$-and $y$-directions on the scan plane. If we assume at least paraxial propagation for the optical axes, then for the reference beam, we still expect that over the scan plane the non-phase terms in Eq. 4 will be slowly varying, and only the phase of the cosine term will be significantly affected by the offsets.

Thus, since as before the origins along the optical axes for $z$ and $z^{\prime}$ are arbitrary, we therefore (for the purposes of the analysis) set $z=0$ and $z^{\prime}=0$ at the scan plane, rather than where the beam optical axes intersect. Therefore, again assuming that the reference beam is travelling at an angle $\theta$ with respect to the object beam, and including the small offsets between the corresponding origins of $x^{\prime}$ and $x$ and $y^{\prime}$ and $y$ with $\delta x=a$ and $\delta y=b$, we can use that $x^{\prime}=x \cos \theta+a, y^{\prime}=y+b$, and $z^{\prime}=-x \sin \theta$ in Eq. 3. The argument of the cosine interference term $\cos (\chi)$ in Eq. 4 then becomes (at the scan plane) a more complicated expression

$$
\cos (\chi(x, y))=\cos \left(-k x \sin \theta-\frac{k}{2}\left(\frac{x^{2}}{R_{0}}-\frac{(x \cos \theta+a)^{2}}{R_{r e f}}\right)-\frac{k}{2}\left(\frac{y^{2}}{R_{0}}-\frac{(y+b)^{2}}{R_{r e f}}\right)+\Delta \phi\right) .
$$

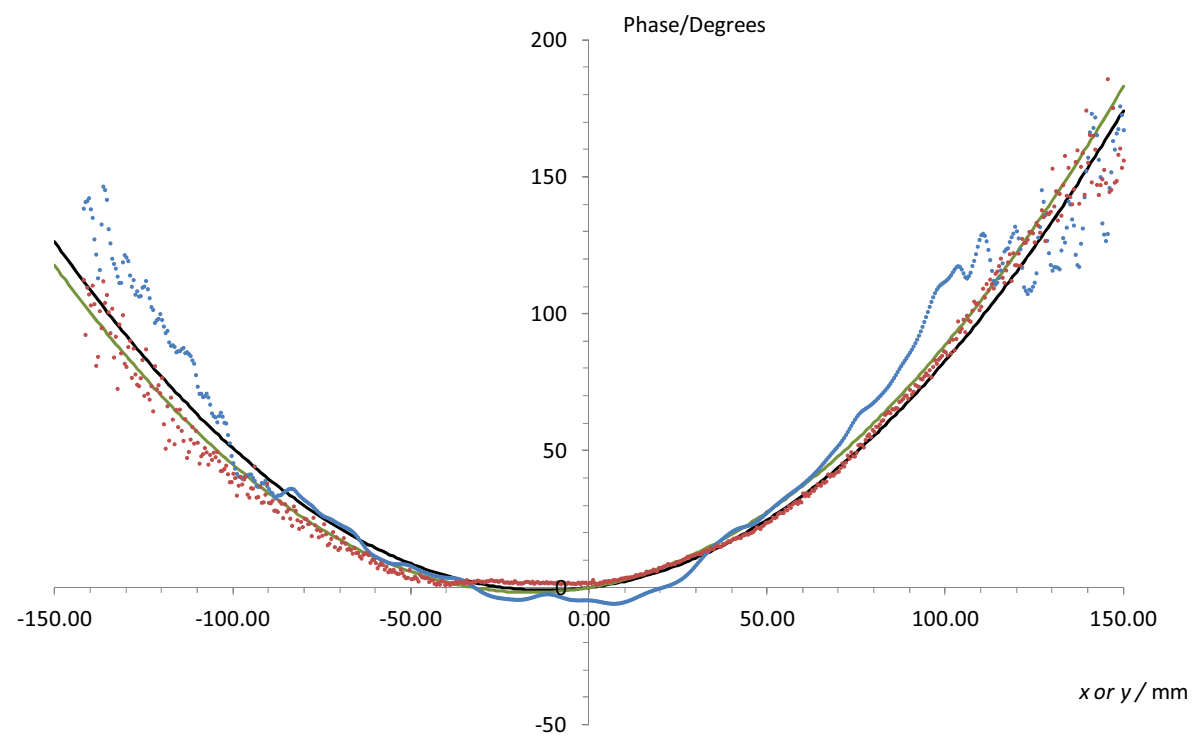

Fig. 6 Best-fit spherical wavefront to the phase difference surface between HUT and corrugated horn. The two sets of data are the averages for a large number of cuts in the $x$-direction (higher curvature) and the $y$-direction (lower curvature). The darker and lighter lines respectively are cuts in the $x$ - and $y$-directions of the best-fit spherical surface 
However, we can clearly rearrange the terms so that the dependencies on $x$ and $y$ can be rewritten in the form

$$
-\chi(x, y)=k x \sin \theta+\frac{k}{2} \frac{\left(x-a^{\prime}\right)^{2}}{R_{x}}+\frac{k}{2} \frac{\left(y-b^{\prime}\right)^{2}}{R_{y}}+\gamma(x, y),
$$

where $a^{\prime}$ and $b^{\prime}$ depend on $R_{r e f}, R_{0}$ and $\cos \theta$ as well as $a$ and $b$. However, we can recover the curvatures $1 / R_{x}$ and $1 / R_{y}$ without needing to locate the symmetry axes $\left(y^{\prime \prime}=y-b^{\prime}=0\right.$ or $x^{\prime \prime}=x$ $-a^{\prime}$ ), since all we need is the $\Delta y_{n}$ when recovering curvatures using the direct fringe spacing method, while for the holographic reconstruction method, we can fit a displaced spherical surface (as was the case in fact, as seen in Fig. 6). Thus, we can still recover the $\Delta(1 / R)$ as before to determine the phase center position.

In the case of the specially designed profiled horn we have considered, it was actually possible to compare the result obtained experimentally with the theoretically predicted phase center and verify the modeling methodology (the horn was designed using mode matching software). As can be seen in Fig. 7, in the far-field, the phase error is not precisely a quadratic term (the phase error being defined as the difference between the predicted equiphase front for the horn in the far-field and a spherical wave emanating from the center of its aperture). The exact location of the phase center thus varies somewhat depending on the amount of the beam over which it is viewed. The analysis of the phase error of the theoretically predicted beam pattern gives a phase center about $18.5 \mathrm{~mm}$ behind the aperture, when fitted with a spherical wave over the main beam to the $1 / e^{2}$ level $(-8.7 \mathrm{~dB})$, see the discussion above in Section 1 . Since the depth of focus of the horn effectively implies an uncertainty in the phase center also of the order of $2.3 \lambda=7 \mathrm{~mm}$, we see that the theoretical position for the phase center of (18.5 $\pm 7.0) \mathrm{mm}$ agrees well with the two experimental predictions of $(16.2 \pm 8.4) \mathrm{mm}$ and $(18.7$ $\pm 9.5) \mathrm{mm}$. This clearly demonstrates the usefulness of both the direct fringe analysis method and holographic reconstruction approach taken in this paper.

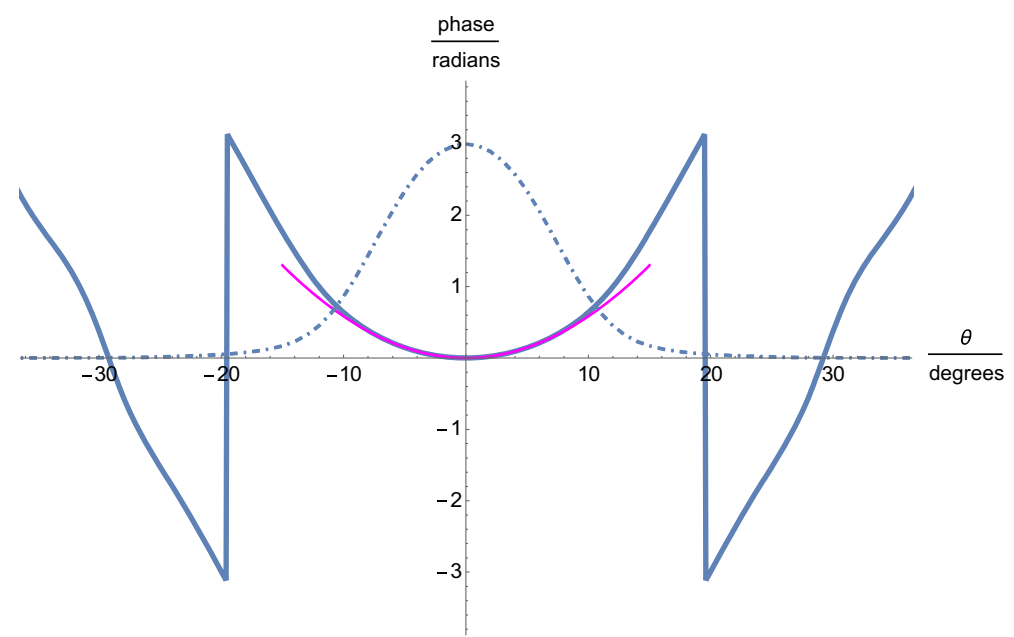

Fig. 7 Far field phase error with respect to a spherical wave emanating from the center of the horn aperture as theoretically predicted, and the best fit spherical phase front error over the main beam. Superimposed is a (linear) plot in intensity of the theoretically predicted beam 


\section{Discussion and Conclusion}

We have presented a technique for phase center retrieval using a holographic interference approach that is applicable to antenna feeds in the millimeter-wave parts of the EM spectrum. The sensitivity of the approach is limited ultimately by the scatter in the data associated with the location of the minima in the cuts across the holograms or the recovery of the distorted object beam phase fronts. In the example discussed in this paper, this led to an error of about $20 \%$ in the determination of the $\Delta z$, which was just tolerable at millimeter wavelengths. Improvements in the detector sensitivity and resulting better signal to noise, as well as an increase in the fringe contrast, should also provide higher quality holograms from which to recover the required fringe curvatures with higher accuracy. This would be particularly essential if the technique is to be applied at higher frequencies in the submillimeter band.

One of the advantages of the technique is it is inexpensive in comparison to the use of a vector network analyzer, for example. Furthermore, if the holographic inference patterns are recorded with sufficient sensitivity, then as well as recovering the phase center positions, the technique could also be used to predict the full far-field phase and amplitude patterns of the horns, including sidelobes, which would be useful for high efficiency coupling to reflector antennas, or for injection into quasi-optical wave-guides. This would also require better definition of the reference beam itself, as this would be useful in the holographic reconstruction. Aberrations in the reference beam do also occur because of the use of an off-axis reflector to deflect the reference beam towards the scan plane. This may explain the asymmetry in the quality of the data in for example Fig. 4a. There is also the possibility of using the technique for the recovery of the phase centers of other types of feed antennas, such as planar lens antennas, which are often difficult to analyze theoretically in a reliable way.

Acknowledgments The authors would like to acknowledge the financial support of the European Space Agency, Enterprise Ireland and the Irish Research Council for the work described in this paper.

\section{References}

1. P.K. Rastogi, E. Hack, editors, Phase Estimation in Optical Interferometry, CRC Press, 2014

2. G. Rajshekhar and P. Rastogi, "Phase estimation using a state-space approach based method," Optics and Lasers in Engineering, 51, 1004-1007 (2013)

3. J. Zela, K. Hoffmann and P. Hudec, "Generalized Approach for Phase Interferometric Measurements of Electromagnetic Field,” PIERS Online, Vol. 3, No. 8, 1340, 2007.

4. A. Miks, and J. Novak, "Phase-shifting microwave interferometry for nondestructive testing," Proc. SPIE 6158, Lightmetry and Light and Optics in Biomedicine 2004, 61580H (April, 2006)

5. I. Hiroshi, T. Shimozuma, M.A. Shapiro, T. Notake, S. Kubo, and R.J. Temkin, "Experimental Verification of Phase Retrieval of Quasi-Optical Millimeter-Wave Beams, ” IEEE Trans. Microw. Theory Tech.,Vol. 54, No.11, 3899-3904, 2006.

6. P. Hariharan, in Holographic Interferometry, ed. by P.K. Rastogi (Springer, Berlin Heidelberg, 1994), p. 7-32.

7. R.J. Mahon, J.A. Murphy, and W. Lanigan, "Digital holography at millimetre wavelengths," Optics Communications, 260, 469-473 (2006)

8. I. McAuley, J. A. Murphy, N. Trappe, R. Mahon, D. McCarthy, and P. McLaughlin, "Applications of holography in the millimeter-wave and terahertz region," Proc SPIE Vol. 7938: Terahertz Technology and Applications IV (February 2011).

9. J.A. Murphy, Ian McAuley, Darragh McCarthy et al. "Quasi-Optical Phase Retrieval of Radiation Patterns of Non-Standard Horn Antennas At Millimetre and Submillimetre Wavelengths," EUCAP-2015, 9th European Conference on Antennas and Propagation, Lisbon, (2015) 
10. Zhang et al. "Terahertz Digital Holography," in Experimental Analysis of Nano and Engineering Materials and Structures, ed. by Gdoutos, E.E. (Ed.) (Springer Netherlands, 2007).

11. M. S. Heimbeck, M. K. Kim, D.A. Gregory and H.O. Everitt, "Terahertz digital holography using angular spectrum and dual wavelength reconstruction methods," Optics Express, VOl. 19, No. 10, 9192-9200, 2011.

12. X. Gao, C. Li, and G. Fang, "Study of image reconstruction for terahertz indirect holography with quasioptics receiver," J. Opt. Soc. Am. A, Vol. 30, No. 6, 1291-1296, 2013.

13. S.-H. Ding, Q. Li, Y.-D.Li and Q. Wang, "Continuous-wave terahertz digital holography by use of a pyroelectric array camera," Opt. Lett., 36, 1993-1995 (2011).

14. Martin Teichman, "Determination of Horn Antenna Phase Centers by Edge Diffraction Theory," IEEE Transactions on Aerospace and Electronic Systems VOL. AES-9, 875-882, 1973.

15. M.A. Teichman, "Precision phase center measurements of horn antennas," IEEE Trans. Antennas and Propagation, vol. 18, September 1970.

16. E.I. Muehldorf, "The phase center of horn antennas" IEEE Trans. Antennas and Propagation, vol. 18, $753-$ 760,1970

17. J.A. Murphy and R. Padman, "Phase centers of horn antennas using Gaussian beam mode analysis," IEEE Trans. Antennas and Propagation, vol-38, 1306-1310, 1990

18. R.J. Wylde, and D.H. Martin, "Gaussian beam-mode analysis and phase-centers of corrugated feed horns," IEEE Transactions on Microwave Theory and Techniques, vol-41, 1691-1699 (1993).

19. P.F. Goldsmith, Quasioptical Systems: Gaussian Beam Quasioptical Propogation and Applications, (IEEE Press, New York, 1998)

20. D. McCarthy, J.A. Murphy, C. O’Sullivan, N. Trappe, M.L. Gradziel, S. Doherty, C. Bracken, Niall Tynan, and Arturo Polegre, "Efficient algorithms for optimising the optical performance of profiled smooth walled horns for future CMB and Far-IR missions," Proc SPIE 9153-42, Astronomical Telescopes and Instrumentation (Montreal 2014).

21. COrE+ The Cosmic Origins Explorer a proposal for ESA's M4 space mission, http://conservancy.umn.edu/ handle/11299/169642 